\title{
Rough Sets and ID3 Rule Learning: Tutorial and Application to Hepatitis Data
}

\author{
D. Tsaptsinos \\ Senior Lecturer, Kingston University \\ School of Mathematics, Penhryn Road \\ Kingston-upon-Thames, Surrey KTI 2EE \\ United Kingdom \\ E-mail: D.Tsaptsinos@kingston.ac.uk
}

\begin{abstract}
In this contribution the basic concepts of rough sets and ID3 using a tutorial-style example are introduced and next the use of the methods for machine learning in the field of hepatitis is addressed. The hepatitis data contains information about 80 patients, expressed using 20 attributes: 19 attributes provide laboratory and clinical examination information and the last attribute defines a binary classification of the patient. The generated results take the form of if...then rules and a brief comparison is given. Finally, the use of rough sets for attribute elimination is introduced.
\end{abstract}

Keywords: data mining, machine learning, rough sets, ID3, medical application, hepatitis

\section{Introduction}

Data mining has come to refer to the process of analysing data and generating new knowledge, hopefully understandable by humans, which was previously hidden and undetected. The overall goal is to create a simplified model of the domain under study. Various techniques for data mining have 
been employed, mostly from the area of inductive learning, with different forms of knowledge representation, for instance weights in an artificial neural network or nodes in a decision tree (IEEE Expert, 1996).

A well known and widely employed inductive learning algorithm is ID3 (Quinlan, 1979, 1986). ID3 and subsequent versions (Quinlan, 1993) base their decisions on statistical measures of the information entropy and knowledge is represented as a decision tree which may easily be converted to a set of rules. An alternative method is based on rough sets which is based on the theory of sets and topology. Rough set theory was introduced by Pawlak (Pawlak, 1982) and since then a number of applications have been reported in diverse fields such as medical diagnosis, conflict analysis and process control (Slowinski, 1992). Rough sets can be used for the purpose of generating if...then rules and/or as a technique for eliminating redundant information prior to use of, say, artificial neural networks.

In the next sections of the paper a simple tutorial is given to introduce various definitions and concepts, next the results obtained using the medical hepatitis data and a brief introduction of the relationship of rough sets and ID3 are presented, and finally the use of rough sets for eliminating attributes is discussed.

\section{Rough Sets Brief Introduction}

The rough set theory extends the classical concept of the membership of an object to a set. Rather than using the classical approach in which either an object belongs to a set or not with rough sets an object may have one of the following properties: Certainly in set, possibly in set and certainly-not in set. Initial data is given using an information system in tabular form of the form Object-->Attribute Value, with a finite set of objects, attributes and a special attribute which provides the class or decision associated with a particular object. Hence, given a subset $X$ of the universe of objects $U$ then we can say that $\chi \in U$ has a certain property $P$ if $\chi \in$ lower approximation of $X$, or that possibly has the property $P$ if $\chi \in$ upper approximation of $X$, or 
that definitely does not have the property $P$ if $\chi \in U$ Ulupper approximation. The definition and how to calculate lower and upper approximation now follows.

\subsection{A Simple Example Illustration}

In this section a simple example is employed to illustrate the basic definitions and concepts of the rough set theory in terms of inductive learning.

Table 1 shows the algorithmic approach for the induction of rules using rough sets.

\section{Table 1. Rough Set Algorithm \\ Initialise universe of objects begin \\ Select decision class \\ Find class relation \\ repeat}

For each attribute do

begin

Select attribute

Find equivalence relation

Find lower subset

Find upper subset

Calculate discriminant inder

end

Select attribute with highest discriminant inder

Generate rules

Reduce universe of objects

Reduce class relation

until no objects with selected decision class end 
Each step of the algorithm is illustrated using the decision table in Table 2. Table 2 contains a universe of objects with 8 objects (u1, u2, u3, u4, u5, u6, u7, u8), each object being described by 3 attributes (Furry, Age, Size) and a class which can take two possible values (either lion or not-lion ).

\begin{tabular}{|l|l|l|l|l|}
\hline \multicolumn{5}{|c|}{ Table 2. A simple decision table } \\
\hline Object & \multicolumn{4}{|c|}{ Attributes } \\
& \multicolumn{5}{|c|}{ Class } \\
\hline & Furry & Age & Size & \\
\hline u1 & furry & old & large & lion \\
\hline u2 & not-furry & young & large & not-lion \\
\hline u3 & furry & young & medium & lion \\
\hline u4 & furry & old & small & not-lion \\
\hline u5 & furry & young & small & not-lion \\
\hline u6 & furry & young & large & lion \\
\hline u7 & not-furry & young & small & not-lion \\
\hline u8 & not-furry & old & large & not-lion \\
\hline
\end{tabular}

Initialise universe of objects: The universe of objects, denoted by $U$ is the set containing all objects (i.e., all row-id's) of the decision table.

$$
\mathrm{U}=\{u 1, u 2, u 3, u 4, u 5, u 6, u 7, u 8\}
$$

Select decision class: $\mathbf{D}$ is a set which contains all possible values of the decision class. In our case $\mathbf{D}=\{$ lion, not - lion $\}$ and the user selects in turn one of the two decision classes.

Find class relation: A class relation is the union of all objects which have the same class. Assuming that the 'lion' class was chosen in the previous step then $\mathbf{Y}_{c}=\mathbf{Y}_{\text {lion }}=\{u 1, u 3, u 6\}$

Find equivalence relation: An equivalence relation is the union of all objects which for a selected attribute $A$ (or attributes) have the same pre- 
defined attribute value $\boldsymbol{v}$ (or values). An equivalence relation is denoted by $\mathbf{R}_{v}(A)$. For example, if the selected attribute is Furry and $v=$ not-furry the equivalence relation is $\mathbf{R}_{\text {not-fumy }}($ Furry $)=\{u 2, u 7, u 8\}$ since objects u2, u7, and u8 have a common value not-furry for attribute Furry. Table 3 shows the equivalence relation for all attributes/value pairs.

\begin{tabular}{|l|l|l|}
\hline \multicolumn{3}{|c|}{$\begin{array}{r}\text { Table 3. Equivalence relations for each } \\
\text { attribute/value combination }\end{array}$} \\
\hline Attribute & Value & Equivalence Relation \\
\hline Furry & furry & $\{\mathrm{u} 1, \mathrm{u} 3, \mathrm{u} 4, \mathrm{u} 5, \mathrm{u} 6\}$ \\
\hline Furry & not-furry & $\{\mathrm{u} 2, \mathrm{u} 7, \mathrm{u} 8\}$ \\
\hline Age & old & $\{\mathrm{u} 1, \mathrm{u} 4, \mathrm{u} 8\}$ \\
\hline Age & young & $\{\mathrm{u} 2, \mathrm{u} 3, \mathrm{u} 5, \mathrm{u} 6, \mathrm{u} 7\}$ \\
\hline Size & large & $\{\mathrm{u} 1, \mathrm{u} 2, \mathrm{u} 6, \mathrm{u} 8\}$ \\
\hline Size & medium & $\{\mathrm{u} 3\}$ \\
\hline Size & small & $\{\mathrm{u} 4, \mathrm{u} 5, \mathrm{u} 7\}$ \\
\hline
\end{tabular}

Find the lower subset: The lower subset is the union of all equivalence relations of the current attribute which are subsets of the class relation. Using set notation this is denoted as

$$
\bigcup\left\{R_{v}(A) \subseteq Y_{c}\right\}
$$

Hence, for each equivalence relation shown in the third column of Table 3 , the question is asked if it is a subset of the selected class relation (lion). It is easy to see that for Size the lower subset, denoted by $\underline{\mathbf{B}}$, is $\{u 3\}$. Similarly, $\underline{\mathbf{B}}($ Furry $)=\varnothing$ and $\underline{\mathbf{B}}($ Age $)=\varnothing$. Therefore, all objects that are included in the lower set can be assigned with certainty to the selected class. 
Find the upper subset: The upper subset is the union of all equivalence relations of the current attribute which when intersected with the class relation give a non-empty set, that is if at least one element of an equivalence relation belongs to the class relation.

$$
\bigcup\left\{R_{v}(A) \cap Y_{c} \neq \varnothing\right\}
$$

Hence, for each equivalence relation shown in the third column of Table 3 , the question is asked if its intersection with the selected class relation (lion) is the empty set or not. If it is not then the equivalence relation is part of the upper subset. Again, for Size the upper subset, denoted by $\overline{\mathbf{B}}$, is \{ul, u2, u3, u6, u8\}.

Similarly, $\quad \overline{\mathrm{B}}(F u r r y)=\{u 1, u 3, u 4, u 5, u 6\}$ and $\quad \overline{\mathrm{B}}($ Age $)=$ $\{u 1, u 2, u 3, u 4, u \delta, u 6, u 7, u 8\}$. Therefore, all objects that are included in the upper set can probably be assigned to the selected class.

Calculate discriminant index: The discriminant index, denoted by $\alpha$, is calculated using the following formula:

$$
\alpha=1-\frac{|\operatorname{card} \overline{\mathbf{B}}(A)-\operatorname{card} \underline{\mathbf{B}}(A)|}{\operatorname{card} \mathbf{U}}
$$

Obviously $0<\alpha<1$. If $\alpha$ is 1 indicates that the objects can be assigned with certainty to the class. If $\alpha$ is 0 indicates that an object(s) can not be assigned to the class. If $\alpha$ is between 0 and 1 means that an object(s) can be partially assigned to a class with $\alpha$ certainty. Calculating $\alpha$, using the lower and upper subsets calculated previously, for the three attributes, we obtain $0.375,0.0$, and 0.5 respectively.

Generate rules: The description of the lower subset of the attribute with the highest discriminant index provides the rules. In our example, attribute Size has the highest index and using the lower subset $\underline{B}($ Size $)=\{u 3\}$ the following rules can be generated: if Size has the value medium then class is lion.

Reduce universe of objects: The universe of objects is reduced using the following formula: 


$$
\mathbf{U}^{\text {new }}=\mathbf{U}-[(\mathbf{U}-\overline{\mathbf{B}}(A) \cup \underline{\mathbf{B}}(A))]
$$

Reduce class relation: The class relation is reduced using the following formula:

$$
\mathbf{Y}_{c}^{\text {new }}=\mathbf{Y}_{c}-\underline{\mathbf{B}}(A)
$$

\section{3. - ID3 Brief Introduction}

The ID3 algorithm implements a scheme for top-down induction of decision trees using depth-first search. The input to the algorithm is a tabular set of training objects, each characterised by a fixed number of attributes and a class designation. To illustrate the operation of ID3 consider the set of training objects of Table 2. Each object is described, as before, by three attributes: Furry (furry, not-furry), Age (old, young) and Size (large, medium, small) and a class designation (lion, not-lion). The output of the algorithm is a decision tree which may easily be translated to a set of rules. The decision-tree for the training objects of Table $\mathbf{2}$ is shown in Figure 1.

The algorithm begins with an empty decision tree and iteratively builds the tree by adding decision nodes, decision branches and terminal nodes. On decision nodes are attributes, on terminal nodes are class-values and on decision branches are attribute values. The ID3 algorithm proceeds as follows:

1. If all objects are of the same class then a terminal node is created and is given the label of the class. In our case, Table 2 objects belong to different classes therefore step 2 is taken

2. The most informative attribute is selected and a decision node together with decision branches are created. The number of decision branches is equal to the number of values the selected attribute takes. In order to find the most informative attribute ID3 uses the information concept of entropy and selects the attribute with the smallest entropy (Shannon and Weaver 1972). Consider attribute Size. Then Figure 1 shows the 


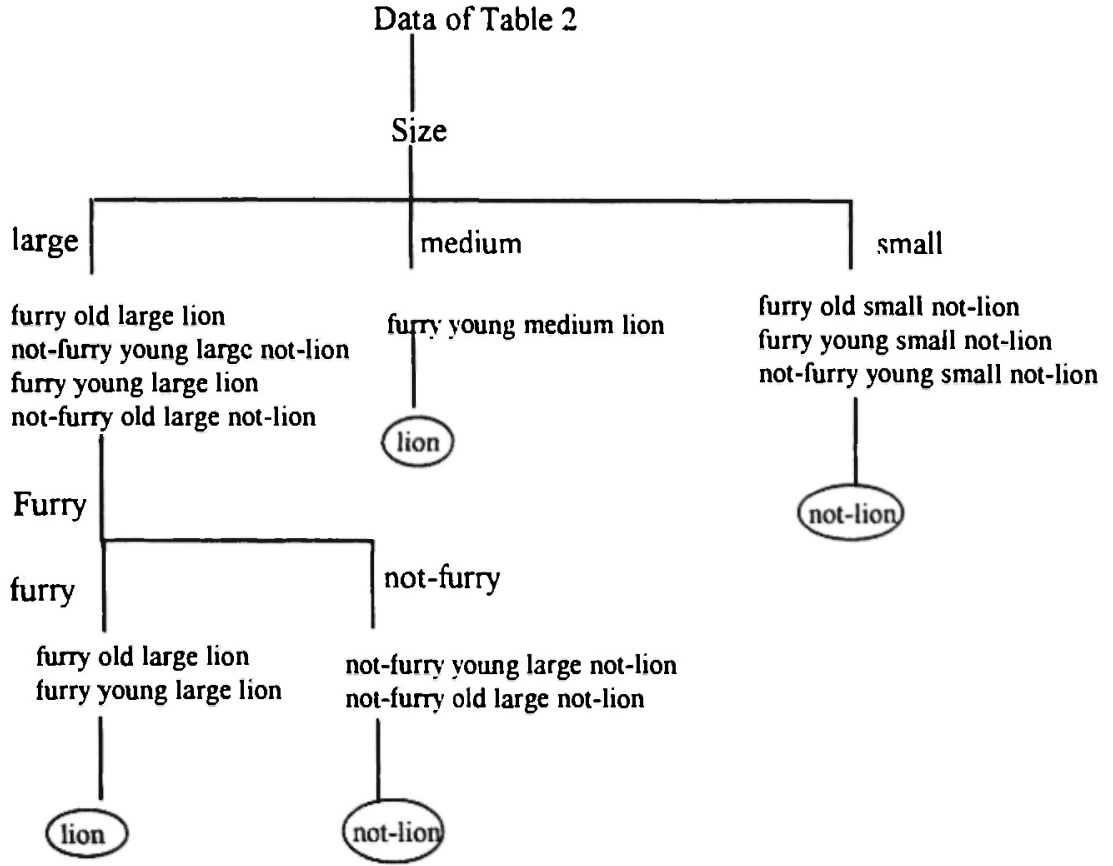

Figure 1: Decision Tree generated using ID3 for data of Table 2

proposed generation of decision node (Size) and decision branches (three in total; large, medium, small). Each decision branch shows the splitting of the objects according to the value of Size. The entropy of Size is calculated using $\sum_{i} w_{1} E_{1}$ where $\mathrm{W}_{\mathrm{i}}$ is defined as the number of objects at the branch $i$ divided by the total number of objects at the parent node; $E_{1}$ is the entropy of each branch and is given by $E_{i}=-\sum_{j} p_{j} \log _{2} p_{j}$ where $p_{j}$ is defined as the number of objects at branch $i$ which have class $j$ divided by the total number of objects in branch $i$. Hence, for branch $i=$ large, medium, small $w_{1}$ is $4 / 8,1 / 8$ and $3 / 8$ respectively. The entropy for each branch is $E_{\text {large }}=-\left(2 / 4 \log _{2} 2 / 4+2 / 4 \log _{2} 2 / 4\right), \quad E_{\text {modurn }}=$ $\left(1 / 1 \log _{2} 1 / 1+0 / 1 \log _{2} 0 / 1\right), E_{\text {mall }}=-\left(0 / 3 \log _{2} 0 / 3+3 / 3 \log _{2} 3 / 3\right)$ respectively.

The total entropy of Size is $4 / 8 * \mathrm{E}_{\text {large }}+1 / 8 * \mathrm{E}_{\text {modium }}+3 / 8 * \mathrm{E}_{\text {mall }}=0.5$. Similarly, the entropy for attributes Furry and Age are 0.607 and 0.955 respectively. Therefore Size is chosen since it has the lowest entropy. 
3. Steps 1 and 2 are applied to each of the new decision branches. In our example for the branches Size $=$ medium and Size $=$ small a terminal node is created since all objects are of the same class in both cases (the labels lion and not-lion are used respectively). For the branch Size=large step 2 has to be applied and the attribute Furry is selected. Both the two new condition branches result in terminal nodes.

\section{Application to Hepatitis Data}

The medical database used is for patients with hepatitis problems. The term hepatitis is used to describe a broad category of clinical and pathological conditions that result from the damage caused to the liver by viral, toxic, pharmacological or immune-mediated attacks. This database is in public domain and can be found at the UCI repository of machine learning databases and domain theories (Murphy and Aha 1994). The original database consists of 155 examples but it was reduced to 80 by removing all examples with a missing attribute value. Each example (object) is described by 19 attributes plus a binary class (Die, Live). Fourteen of the attributes give binary information (yes, no) whereas the rest of the attributes were originally continuous but they were discretised according to boundary values as advised by the donor of the data set. Each continuous attribute has between 6 to 10 of discretised ranges.

Following the algorithm for the class relation Live the discriminant indices for all attributes were generated as:

Hence, the attribute PROTIME was selected and 4 rules were generated from each equivalent relation of the lower set:

if PROTIME is $<10$ then Class is Live

if PROTIME is $[=>50-<60]$ then Class is Live

if PROTIME is $[=>70-<80]$ then Class is Live

if PROTIME is $[=>80-<90]$ then Class is Live 
Tutorial and Application to Hepatitis Data

\begin{tabular}{|c|c|}
\hline \multicolumn{2}{|c|}{ Table 4. Hepatitis data } \\
\hline Attribute Name & Attribute values/Occurrences \\
\hline Age & $\begin{array}{l}(20-<30) / 11 ; \quad(30-<40) / 32 ; \quad(40- \\
<50) / 17 ; \quad(50-<60) / 15 ; \quad(60-<70) / 4 ; \\
(\Leftrightarrow>70) / 4\end{array}$ \\
\hline Sex & Male/69; Female/11 \\
\hline Steroid & No/38; Yes/42 \\
\hline Antivirals & No/21; Yes/59 \\
\hline Fatique & $\mathrm{No} / 52 ; \mathrm{Yes} / 28$ \\
\hline Malaise & No/31; Yes/49 \\
\hline Anorexia & $\mathrm{No} / 12 ; \mathrm{Yes} / 68$ \\
\hline Liver Big & No/13; Yes/67 \\
\hline Liver Firm & $\mathrm{No} / 38 ; \mathrm{Yes} / 42$ \\
\hline Spleen palpable & No/15; Yes/65 \\
\hline Spiders & $\mathrm{No} / 25 ; \mathrm{Yes} / 55$ \\
\hline Ascites & $\mathrm{No} / 12 ; \mathrm{Yes} / 68$ \\
\hline Varices & No/10; Yes/70 \\
\hline Bilirubin & $\begin{array}{l}(0-<0.39) / 1 ; \quad(0.39-<0.8) / 24 ; \quad(0.8- \\
<1.2) / 26 ; \quad(1.2-<2) / 25 ; \quad(2-<3) / 5 ; \\
(\Rightarrow 3) / 3\end{array}$ \\
\hline Alk phosphate & $\begin{array}{l}(0-<33) / 2 ; \quad(0.33-<80) / 29 ; \quad(80- \\
<120) / 25 ; \quad(120-<160) / 10 ; \quad(160- \\
<200) / 9 ; \quad(200-<250) / 4 ; \quad(=>250) / 1\end{array}$ \\
\hline Sgot & $\begin{array}{l}(13-<100) / 60 ; \quad(100-<200) / 14 ; \\
(200-<300) / 5 ;(300-<400) / 0 ; \quad(400- \\
<500) / 1 ;(=>500) / 0\end{array}$ \\
\hline Albumin & $\begin{array}{l}(2.1-<3) / 8 ; \quad(3-<3.8) / 16 ; \quad(3.8- \\
<4.5) / 12 ; \quad(4.5-<5) / 43 ; \quad(5-<6) / 1 ; \\
(=>6) / 0\end{array}$ \\
\hline Protime & $\begin{array}{l}(0-<10) / 1 ; \quad(10-<20) / 0 ; \quad(20-<30) / 4 ; \\
(30-<40) / 9 ; \quad(40-<50) / 10 ; \quad(50- \\
<60) / 13 ;(60-<70) / 12 ;(70-<80) / 12 ; \\
(80-<90) / 5 ; \quad(=>90) / 14\end{array}$ \\
\hline Histology & No/47; Yes/33 \\
\hline Class & Die/13; Live/67 \\
\hline
\end{tabular}




\begin{tabular}{|l|l|}
\hline \multicolumn{2}{|c|}{ Table 5. Discriminant indices (first pass) } \\
\hline Attribute & Discriminant index \\
\hline Age & 0.2 \\
\hline Sex & 0.14 \\
\hline Steroid & 0.0 \\
\hline Antivirals & 0.0 \\
\hline Fatique & 0.0 \\
\hline Malaise & 0.0 \\
\hline Anorexia & 0.15 \\
\hline Liver Big & 0.16 \\
\hline Liver Firm & 0.0 \\
\hline Spleen Palable & 0.0 \\
\hline Spiders & 0.0 \\
\hline Ascites & 0.0 \\
\hline Varices & 0.0 \\
\hline Bilirubin & 0.02 \\
\hline Alk Phosphate & 0.04 \\
\hline SGOT & 0.08 \\
\hline Albumin & 0.09 \\
\hline Protime & 0.39 \\
\hline Histology & 0.0 \\
\hline & \\
\hline
\end{tabular}

Then the size of the decision table was reduced and the class relation for Live was recalculated. The second pass considered attribute pairs (PROTIME, other attribute) and the discriminant indices were calculated as shown below: 


\begin{tabular}{|l|l|}
\hline \multicolumn{2}{|c|}{ Table 6. Discriminant indices (second pass) } \\
\hline Attribute Pairs & Discriminant index \\
\hline Protime, Age & 0.55 \\
\hline Protime, Sex & 0.12 \\
\hline Protime, Steroid & 0.41 \\
\hline Protime, Antivirals & 0.16 \\
\hline Protime, Fatique & 0.33 \\
\hline Protime, Malaise & 0.14 \\
\hline Protime, Anorexia & 0.16 \\
\hline Protime, Liver Big & 0.16 \\
\hline Protime, Liver Small & 0.37 \\
\hline Protime, Spleen & 0.04 \\
\hline Protime, Spiders & 0.35 \\
\hline Protime, Ascites & 0.14 \\
\hline Protime, Varices & 0.35 \\
\hline Protime, Bilirubin & 0.55 \\
\hline Protime, Alk Phosphate & 0.73 \\
\hline Protime, SGOT & 0.24 \\
\hline Protime, Albumin & 0.27 \\
\hline Protime, Histology & 0.57 \\
\hline
\end{tabular}

Hence, at the end of the second pass for the class Live, the pair of attributes PROTIME and HISTOLOGY were selected and 4 further rules were generated:

if PROTIME is [ $=>20-<30]$ and HISTOLOGY is no then class is Live if PROTIME is $[=>40-<50]$ and HISTOLOGY is no then class is Live if PROTIME is $[=>60-<70]$ and HISTOLOGY is no then class is Live if PROTIME is [ $=>90]$ and HISTOLOGY is no then class is Live

For the third pass, again reducing the size of the decision table and class relation, triplets of attributes were considered (PROTIME, HISTOLOGY, 
other attribute) and ALK-PHOSPHATE had the highest discriminant index. The third pass generated 9 more rules involving PROTIME, HISTOLOGY, and ALK-PHOSPHATE:

if PROTIME is $[=>40-<50]$ and HISTOLOGY is yes and ALK is $[=>200-$ $<250]$ then Class is Live if PROTIME is $[=>40-<50]$ and HISTOLOGY is no and $A L K$ is $[\Rightarrow 33-$ $<80]$ then Class is Live if PROTIME is $[=>40-<50]$ and HISTOLOGY is no and $A L K$ is $[=>160-$ $<200]$ then Class is Live if PROTIME is $[=>60-<70]$ and HISTOLOGY is yes and ALK is $[=>33-$ $<80]$ then Class is Live

if PROTIME is [ $=>90]$ and HISTOLOGY is yes and ALK is $[=>33-<80]$ then Class is Live

if PROTIME is $[=>60-<70]$ and HISTOLOGY is yes and ALK is $[=>120$ $<160]$ then Class is Live

if PROTIME is [ $=>90]$ and HISTOLOGY is yes and ALK is $[=>120-<160]$ then Class is Live

if PROTIME is [ $=>60-<70]$ and HISTOLOGY is yes and ALK is [ $\because>160-$ $<200]$ then Class is Live

if PROTIME is [ $=>20-<30]$ and HISTOLOGY is yes and ALK is $[=>160-$ $<200]$ then Class is Live

Finally, the fourth and final pass for the Live class generated 2 more rules considering the three previous attributes plus STEROID (Ascites or Albumin could have been selected as well).

The two new rules were as follows:

if PROTIME is $[=>60-<70]$ and HISTOLOGY is yes and ALK is $[=>80-$ $<120]$ and STEROID is no then class is Live if PROTIME is $[=>40-<50]$ and HISTOLOGY is yes and ALK is $[=>80-$ $<120]$ and STEROID is no then class is Live

The same procedure was followed for the other class but the results are not reported here. 
The same database was employed using the ID3 algorithm and Table 7 shows the results of a brief comparison of the two methods. An important observation made is that the top level rules agreed.

\begin{tabular}{|l|l|l|}
\hline \multicolumn{3}{|c|}{ Table 7. A brief comparison of rough sets and $\mathrm{ID3}$} \\
(only for Class=Live) \\
\hline Criterion & Rough Sets & $\mathrm{D3}$ \\
\hline Number of rules & 19 & 18 \\
\hline Number of conditions & 46 & 33 \\
\hline $\begin{array}{l}\text { Number of attributes } \\
\text { used }\end{array}$ & $\begin{array}{l}4 \text { (protime, histology, } \\
\text { alk-phosphate, steroid) }\end{array}$ & $\begin{array}{l}5 \text { (protime, histology, } \\
\text { alk-phosphate, age, } \\
\text { spiders) }\end{array}$ \\
\hline $\begin{array}{l}\text { Number of identical } \\
\text { rules }\end{array}$ & 5 & \\
\hline
\end{tabular}

\section{Reducts}

An alternative use of the rough set theory is to remove superfluous attributes, thus to reduce the original set of attributes by selecting a subset of the original attributes without losing information. A point to notice is that unlike databases, the tabular information system analysed using rough sets may contain duplicate tuples. The reduced set of attributes can be employed for decision-making using rough sets as illustrated in the previous sections or can be thought of as a pre-processing stop for, say, artificial neural networks.

Reducts can be calculated using the distinction table, first introduced by Wroblewski (Wroblewski 1995), and the procedure is as follows:

- Create a table with each row comparing two objects. For each attribute where the two objects have different attribute values enter 1 , otherwise 0 . To illustrate the rule consider the following table: 


\begin{tabular}{|c|c|c|c|}
\hline Object & Attribute-A & Attribute-B & Attribute-C \\
\hline $\mathrm{u} 1$ & alpha & 5 & less \\
\hline $\mathrm{u} 2$ & alpha & 4 & less \\
\hline $\mathrm{u} 3$ & beta & 4 & more \\
\hline $\mathrm{u} 4$ & gamma & 5 & less \\
\hline
\end{tabular}

Thus comparing objects $u l$ and $u 2$ results to 010 since they agree on attributes $\mathrm{A}$ and $\mathrm{C}$ but differ on attribute $\mathrm{B}$. Continuing in a similar way the following distinction table is produced:

\begin{tabular}{|c|c|}
\hline Object, Object & Comparison of Attributes \\
\hline $\mathrm{u} 1, \mathrm{u} 2$ & 010 \\
\hline $\mathrm{u} 1, \mathrm{u} 3$ & 111 \\
\hline $\mathrm{u} 1, \mathrm{u} 4$ & 100 \\
\hline $\mathrm{u} 2, \mathrm{u3}$ & 101 \\
\hline $\mathrm{u} 2, \mathrm{u4}$ & 110 \\
\hline $\mathrm{u3}, \mathrm{u4}$ & 111 \\
\hline
\end{tabular}

- To find the reduct(s) (i.e., the minimal subset of columns) all possible combinations that the comparison of attributes can take are created. Then the combination(s) which satisfies the following rule is found: for all (object, object) the entry in the comparison-of-attributes column must have at least one value of $I$ as in the combination under consideration (not necessarily the same position for all entries). The rule is applied first to combination(s) with a single 1 and then applied for all candidates with $2,3, \ldots$ attributes equal to 1 . A number of reducts might be found but supersets are obviously rejected.

Continuing with the trivial decision table shown above, all possible combinations are as follows: 


\begin{tabular}{|c|}
\hline Possible Combinations \\
\hline 001 \\
\hline 010 \\
\hline 011 \\
\hline 100 \\
\hline 101 \\
\hline 110 \\
\hline 000 (ignored) \\
\hline 111 (ignored) \\
\hline
\end{tabular}

The first combination to be considered is 001 . The combination has a single 1 at position 3 , hence each entry of the comparison of attributes column must have a 1 at position 3 . This is not true for the first entry (010) therefore the next combination is considered and so on. Continuing the same way one can see that the combination of 110 requires every entry of the comparison of results column to have a 1 in position 1 or 2 , which is valid in our example. Hence, a possible reduct is Attribute-A and Attribute-B.

The above procedure gives exact solution which for more complicated problems will be time consuming, hence researchers have looked into the use of genetic algorithms as a method of approximating the solution (Wroblewski 1995).

\section{Evaluation of Rules and Techniques}

From a medical point of view it is necessary to state that because in this paper I did not set out to evaluate the rules created by the methods but to present the methods, the rules should not be considered as medical knowledge. In addition, the methods had at their disposal limited and unrepresentative data (for instance PROTIME has no value for the range 1020. This is an important omission since this range represents what is 
considered to be the normal range). Also the choice of discretisation intervals affect the results and more work is required. Some general comments can be made though:

a) The selected attributes indicate that decision=live depends on laboratory tests rather than clinical examination or symptoms, and

b) The PROTIME (prothrombin time) attribute is considered to be the most useful index of severity and therefore rough sets and ID3 did well to identify PROTIME as the most important attribute.

Now consider the rules employing the usual indices of accuracy and coverage [Holsheimer and Siebes 1994]. Accuracy refers to the proportion of objects that satisfy the conditions of the rule that also satisfy the classification part. All rules generated by both systems have an accuracy of 1. This implies that there are no objects in the training set which given certain conditions from a rule indicate a different classification part. This was expected since both methods generate certain rules, at least in the way the methods are used in this work. The coverage index refers to the proportion of objects that satisfy the prediction of a rule that also satisfy the conditions of the rule. As an example, the rule protime $=[=>70-<80]$ then class=live has a coverage of $18 \%$ since 12 objects out of 67 describing class=live have protime equal to the given condition. The implicit assumption is that the more objects that support a rule exist in the training set the greater the possibility that the rule is valid. Whereas, this is a good indicator it is not clear. Consider the rule protime $=[=>0-<10]$ then class=live which has a coverage of $0.015 \%$ (supported by only one object). Therefore, one might question the validity of the rule but as shown in [Duntsch and Gediga 1996] the above assumption does not hold at every situation. An interesting approach for the evaluation of the generated rules using randomization techniques can be found in Duntsch and Gediga 1996].

Both methods operate on the basis that given a set of classified objects the algorithm will process the objects to formulate a set of rules. Both methods place the responsibility on the developer to select a training set of 
objects in terms of representative attributes, attribute-values and size. Therefore both systems' power is the language defined by the developer to characterise the training set. In general, both methods follow the principle of insufficient reasoning where in the absence of further knowledge all objects are assumed to be equally likely, and they both remove objects that have already being classified. There is a slight difference in the way they select an attribute but it has been shown (Wang et al. 1986) that the rough set criterion is a special case of the entropy criterion used by ID3. In particular, the attribute selection will be the same if objects in every equivalence class that belong to the doubtful regions are distributed equally among the classes.

Another difference is that ID3 considers all classes at each stage whereas the rough sets methodology considers one class at a time until all objects with that class are exhausted. Overall, the rough sets approach is more cautious, if it can not make a decision it would not. Furthermore rough sets can generate additional rules. For instance, in Section 4 it was mentioned that Ascites or Albumin could have been selected instead of Steroid. This can be useful if data for Steroid was missing. This is also true if reducts are used and then one might have an alternative rule to apply.

Overall, Rough sets and ID3 may be compared in terms of handling of noise, time and memory constraints during learning, syntactic and semantic simplicity of results, effect of dynamic databases. Noisy data might take the form of corrupted values and missing attribute values. The latter problem known as the unknown value problem has been attacked by ID3 and Rough Sets workers. Recent versions of ID3 include the ability to handle noise and Rough Sets have the potential for handling noise gracefully. ID3 has a low time complexity whereas Rough Sets especially with the use of reducts has a high time complexity. ID3 sometimes generates not simple rules for humans to evaluate whereas Rough Sets seems to handle this criterion better. Both methods seem to have problems with dynamic databases. If some data is added the whole learning process has to be conducted again. 


\section{Final Remarks}

The ID3 algorithm has been used on a variety of tasks, in the standard or a modified form, and several studies have compared ID3 performance with other techniques [Mooney 1989, Mingers 1987]. ID3 was found to perform similarly to back-propagation neural network and multiple regression. The use of the technique uncovered various deficiencies in the basic mechanism. For example, studies by Kononenko et al. [Kononenko et al. 1984] highlighted the deficiency of favouritism towards attributes with a large number of values. The generation of complex decision trees which are difficult to interpret and therefore check, the possible incorporation of irrelevant attributes into the decision tree, the lack of guarantee that the selected attribute at any stage provides the best decision tree for the following stages are some of other reported deficiencies.

The use of rough sets provides a solid framework for data mining which at the same time is simple and easy to follow. Analysis based on rough set employs only information within the data and does not need additional model assumptions such as prior probabilities or the expression of imprecision using external numerical values. In addition, the use of rough set theory for the induction of knowledge by-pass the so-called bottle-neck problem experienced using the traditional construction of knowledge-based systems and avoids the employment of user-defined confidence degree for a rule. A number of issues which were not addressed in this contribution need further work and research. For instance how to deal with objects with missing values for one or more attributes and the faster generation of reducts.

It is the author's belief that a single learning method cannot overcome all difficulties presented by real-world applications and only the integration of learning methods will enable us to develop better solutions. The interested reader can consult [Yasdi 1995] for an example of such an integration between neural networks and rough sets. 


\section{References}

Holsheimer, $\mathrm{M}$ and Siebes, A. 1994 Data Mining: the search of knowledge in databases. Technical report CS-R9406, CWI Amsterdam

Duntsch, I. and Gediga, G. 1996 Statistical evaluation of rough set dependency analysis, Preprint, http://frege.infj.ulst.ac.uk/papers/ rougheva.html

IEEE Expert, 11, 5, October 1996

Kononenko, I., Bratko, I. and Roskar, E. 1984 Experiments in automatic learning of medical diagnostic rules, Technical Report, Jozaf Stefan Institute, Ljubljana, Yugoslavia

Mingers, J. 1987 Rule induction with statistical data - a comparison with multiple regression, Journal of Operational Research, 38, 4, 347-351.

Mooney, R. 1989. An experimental comparison of symbolic and connectionist learning algorithms, Proceedings $1 I^{\text {th }}$ International Joint Conference on AI (IJCAI-89), Morgan Kaufmann, San Francisco, California, 775-780.

Murphy, P.M. and Aha, D.W. 1994 UCI Repository of machine learning databases, http://www.ics.uci.edu/ mlearn/MLRepository.html, Irvine, CA: University of California, Department of Information and Computer Science

Pawlak, Z. 1982. Rough sets, International Journal of Computer and Information Sciences, 11, 341-356

Quinlan, J.R. 1979. Discovering rules by induction from large collections of examples, in: Expert systems in the microelectronic age, edited by Michie D. , Edinburgh, Edinburgh University Press, 168-201.

Quinlan, J.R 1986. Induction of decision trees, Machine Learning, 1, 81106.

Quinlan, J.R 1993. C4.5: Programs for Machine Learning, San Francisco, Morgan Kaufmann.

Shannon C.E. and Weaver, W. 1972. The Mathematical Theory of Communication, Chicago, University of Illinois Press. 
Slowinski, R. 1992. Intelligent Decision Support: Handbook of Applications and Advances of the Rough Sets Theory, Dordrecht, The Netherlands, Kluwer Academic Publishers.

Wong S.K.M., Ziarko, W. and Li Ye R. 1986. Comparison of rough set and statistical methods in inductive learning, International Journal of ManMachine Studies, 20, 1986, 469-483.

Wroblewski, J. 1995. Finding minimal reducts using genetic algorithm (extended version), Warsaw University of Technology, Institute of Computer Science Technical Report 16/95.

Yasdi, R. 1995. Combining Rough Sets Learning - and Neural Learningmethod to deal with uncertain and imprecise information, Neurocomputing, 7, 1, 61-71. 